\title{
Urban Development Evaluation By Using AHP
}

\author{
Yibin Zhou \\ School of North China Electric Power University, Baoding 071000, China; \\ 2212327403@qq.com
}

Keywords: Analytic Hierarchy Process, Urban Development Evaluation

\begin{abstract}
The world is becoming urbanized rapidly while the demand of urban construction land continues to increase, and the growth of urbanization will persist. Therefore,healthy and stable development of the city is particularly important.In this paper, we take Sochi for example. According to the Analytic Hierarchy Process(AHP),we obtained suitability evaluation weighting coefficient table of suitability evaluation of urban land in land use. And then calculate the indicators of the success rate of urban residents to reflect the success rate of urban development to a certain extent.Finally, using the weights and field evaluation index that we calculate to successfully get index of Sochi city residential land development.
\end{abstract}

\section{Introduction}

With the influx of immigrants into the city, the demend of the urban will continue to increase, the growth of urbanization will persist, The world is rapidly urbanized. Moreover, the spread of the city is not just around the urban built-up area of compact diffusion but rather to the present evolution pattern of more diversified. And we recognized that many nations, developed or developing, the land in most cities around the region are changing rapidly as well as a large number of countryside land has transformed to the national construction land .Urban sprawl contributes to a certain degree of contagion. The contagion of the city has brought many negative effects, which leads to resource waste , environment pollution,a series of social and economic problems .Based on this, we use the AHP to determine the success index of urban land use.

\section{Analytic Hierarchy Process[1][2](AHP)}

According to the residents of the land depends on the geographical environment and human environment, we use the AHP to calculate the corresponding weight of the impact of urban land use in various environments

\section{The two-hierarchy structure}

Considering the development of the city.We use 5 indicators to determine the weight of each index:Terrain condition,Educational resources,Communal facilities,Traffic accessibility and Environmental quality.We construct the two hierarchy structure, which is shown in the following figure.

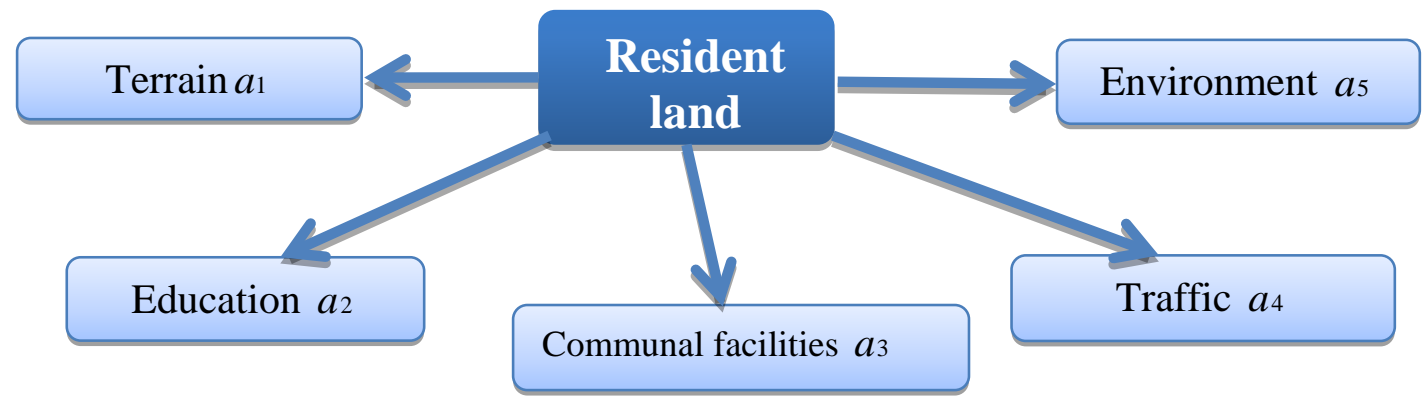

\section{Obtain the index weight:}

Fig. 1 The two hierarchy structure of model

- Establish pairwise comparison matrix. 
Establish pairwise comparison matrix $A=\left(a_{i j}\right)$. Each entry $a_{j k}$ of the matrix represents the importance of the $j^{\text {th }}$ criterion relative to the $k^{\text {th }}$ criterion.

Where: If $a_{j k}>1$, then the $j^{\text {th }}$ criterion is more important than the $k^{\text {th }}$ criterion

if $a_{j k}<1$, then the $j^{\text {th }}$ criterion is less important than the $k^{\text {th }}$ criterion.

If two criteria have the same importance, then the entry ${ }^{a_{j k}}$ is 1.

The entries $a_{j k}$ and $a_{k j}$ satisfy the following constraint:

$$
a_{j k} \times a_{k j}=1
$$

Obviously, $a_{j j}=1$ for all $j$. The relative importance between two criteria is measured according to a numerical scale from 1 to 9 , as shown in Table 1 :

Table 1 Table of relative scores

\begin{tabular}{ll}
\hline Value of $a_{j k}$ & Meaning \\
\hline 1 & $j$ and $k$ are equally important \\
3 & $j$ is slightly more important than $k$ \\
5 & $j$ is more important than $k$ \\
7 & $j$ is strongly more important than $k$ \\
9 & $j$ is absolutely more important than $k$ \\
$2,4,6,8$ & Intermediate values to reflect fuzzy inputs \\
\hline
\end{tabular}

- Calculate the eigenvalues and eigenvector

The greatest eigenvalue of matrix $A$ is $\lambda$ and the corresponding eigenvector is $\omega$ :

$$
A \omega=\lambda \omega
$$

where $A$ is the comparison matrix

- Do the consistency check

The consistence index(CI) and consistence radio(CR) can be calculated by formula(3)

$$
C I=\frac{\lambda-n}{n-1}
$$

Where: $n$ denotes the exponent number of matrix.

$C I$ can be compared with a random matrix $(R I)$, which is shown in Table 2.

Table 2: Values of the Random Index $(R I)$

\begin{tabular}{llllllllllll}
\hline $\mathrm{n}$ & 1 & 2 & 3 & 4 & 5 & 6 & 7 & 8 & 9 & 10 & 11 \\
\hline$R I$ & 0 & 0 & 0.58 & 0.90 & 1.12 & 1.24 & 1.32 & 1.41 & 1.45 & 1.49 & 1.51 \\
\hline
\end{tabular}

The consistence radio(CR) can be calculated by formula (4).

$$
C R=\frac{C I}{R I}
$$

where $C R<0.1$

\section{Result \&Analysis}

According to the data we have collected, we can get the results through the above steps.

$>$ Pairwise comparison matrix : 


$$
A=\left[\begin{array}{lllll}
1 & \frac{1}{4} & \frac{1}{3} & \frac{1}{3} & \frac{1}{3} \\
4 & 1 & \frac{1}{2} & \frac{1}{2} & \frac{1}{3} \\
3 & 2 & 1 & 3 & \frac{1}{2} \\
3 & 2 & \frac{1}{3} & 1 & \frac{1}{3} \\
3 & 3 & 2 & 3 & 1
\end{array}\right]
$$

$>$ Weight vector of criteria hierarchy:

$$
w=\left[\begin{array}{lllll}
0.068 & 0.137 & 0.271 & 0.156 & 0.368
\end{array}\right]^{T}
$$

In this hierarchy, $C R=0.0825<0.1$.

$>$ Index vector of program level:

$$
w^{(1)}=\left[\begin{array}{lllll}
0.6 & 0.78 & 0.82 & 0.87 & 0.89
\end{array}\right]
$$

$>$ We get the combination Success indicators by formula $w_{f}=w^{*}{ }_{w}^{(1)}$

$$
W_{f}=0.83312
$$

At last, we can obtain Sochi city residential land development success indicators:

$$
W_{f}=0.83312
$$

\section{Summary}

Taking into account multiple factors, we calculate the weight of the factors affecting the development of residential land in urban development, we take 5 indicators,fully considering the terrain conditions, educational resources, accessibility, public facilities, environmental quality,finally Sochi city residents with the index of success:

$$
W_{f}=0.83312
$$

According to the indicators of Sochi city residents in the development of success.Moreover, this process can be applied to many other cities.t the same time, the same method can be used to determine the factors that influence the development of urban industrial land.

\section{References}

[1] http://www.dii.unisi.it/\%mocenni/Note_AHP.pdf 